\section{Francomichele Puca Marina de Tommaso}

F. Puca (西) • M. de Tommaso Neurological Clinic I, University of Bari, Policlinico, Piazza Giulio Cesare 11, I-70124 Bari, Italy e-mail: m.detommaso@neurol.uniba.it Tel.: +39-080-5478565 Fax: +39-080-5478565

\title{
The psychophysiological evidence in migraine supports a biobehavioral approach
}

The precise etiopatogenesis of migraine is unclear. A genetic predisposition for migraine is generally accepted to be the cause of low individual thresholds to attacks: the fact that anyone may suffer from one or two migraine attacks in life indicates that the migraine attack in itself is not abnormal but the tendency toward recurrent attacks is [1]. The recurrence and the duration of headache affect the severity of migraine and its chronicity, so the real question in migraine management regards two factors, the first concerning the attack onset and the second the pain maintenance.

Clinical features suggest that migraine may be primarily characterised by a dysfunction of the central nervous system (CNS) [2]. Activation of the trigeminovascular system may cause the attack symptoms. The trigeminal nerve is activated in migraine attacks after a cascade of events including cortical phenomena resembling spreading depression. Furthermore, it is responsible for vascular reactions and, consequently, pain-system involvement [3].

Neurophysiologic studies performed between attacks have revealed a condition of cortical hyperexcitability predisposing to attack onset. For instance, increased amplitude of visual evoked potentials or of event-related potentials (CNV, contingent negative variation) are thought to reflect cortical hyperexcitability in the headache-free interval [4-6]. Many studies measuring visual-, acoustic- and event-related potentials in migraine concur with the hypothesis that the high amplitude of averaged potentials is caused by a phenomenon of deficient habituation, or even potentiation, of the evoked response during stimulus repetition and at increasing intensity, which should be the crucial abnormality in cortical information processing [7, 8]. A "reducing" pattern occurs normally during repetition of the same stimulus and at increasing intensities as a cortical mechanism protecting from sensory overstimulation [9].

Habituation and potentiation of evoked potentials are cortical phenomena modulated by central monoamines, particularly serotonin $[10,11]$, which plays a fundamental role in migraine pathogenesis [12]. The abnormal cortical information processing in migraine may be the result of low activity in serotononergic pathways, but it seems quite reductive to attribute this phenomenon to a biochemical feature without considering it within a complex behaviour predisposing to headache recurrence. In a study on visualevoked potentials and personality features in headache, the potentiation phenomenon was found to correlate with an arousal-related personality trait in migraine patients [13]. 
An abnormal behavioural pattern emerged also when reflexes were evaluated in migraine. In a recent study on blink reflex in migraine, modulation of polysynaptic responses was altered in the sense of a reduced inhibitory effect exerted by attention and habituation to the stimulus [14]. Normally, when a subject pays attention to a potentially dangerous stimulus, he adjusts his defensive reaction to the stimulus quality to obtain the maximal efficiency. In the case of blinking, the attention to the stimulus reduces the intensity and duration of the eye closure reflex, so that the subject does not lose important visual stimulations useful for a correct reaction. When the stimulus is preceded by a warning, the reaction strategy becomes a more effective, purposeful reaction. In migraine patients the lack of inhibition of polysynaptic blink reflex responses by expectancy may be due to a learning disturbance preventing an adequate reflex response to the stimulus. A specific behavioural pattern consisting of a loss of adaptation and hypersensitivity against environmental conditions could be confirmed by these results in migraine.

Abnormalities in the modulation of evoked responses against stimulus features were investigated in relation to personality traits and diagnostic categories of psychiatric disorders and a habituation deficit and potentiation by stimulus intensity were found in subtypes of depression [15, 16]. Recently, investigations into the psychiatric aspects of migraine confirmed the comorbidity of panic disorder and depression [17, 18].

Furthermore, high comorbidity of depressive and anxiety disorders was observed in patients with chronic daily headache and, specifically, transformed migraine [19]. How the behavioural disturbance, probably linked with psychiatric disorders, may be genetically determined or induced by migraine itself is not clear. Studies on childhood migraine found that psychiatric symptoms [20] and neurophysiological abnormalities [21, 22] are clear at the onset of the disease and probably predispose to headache. If the dysfunction in cortical information processing predisposes to attack recurrence, other causes may subtend pain maintenance conditioning the attack duration and migraine chronicity.

Pain experience is profoundly modified by contextual cues, affect, attention and expectancy [23] and, in migraine mechanisms of neuroplasticity induced by nociception, these should be supposed at spinal and probably supraspinal levels. Phenomena of central sensitization involving the brainstem trigeminal neurons and the descending modulatory pain pathways were postulated in headache maintenance during migraine attack [24]: cutaneous allodynia prevails in older patients, probably related to the cumulative number of migraine attacks experienced over the years.

In transformed migraine, a windup phenomenon was suggested on the basis of the temporal summation of the second pain [25]. Recently, we evaluated the cortical responses evoked by laser stimulation, which specifically excite the nociceptive fibers. In transformed migraine, the correlation between the amplitude of laser-evoked potentials and stimulus intensity, clear in normal subjects and in migraine patients, was lost because chronic headache patients showed great cortical potentials even at not painful stimuli delivered at both facial and hand levels [26]. This pattern may be explained by mechanisms of supraspinal sensitization linked with pain-related memory, which were also supposed in other chronic pain syndromes: chronic pain seems to lead to altered processing in associative areas and may contribute to hyperalgesic states in the 
absence of peripheral nociceptive stimulation [27]. These pain memories can also be influenced by psychological processes [28].

All considering, behavioural abnormalities subtend all migraine aspects: they may both pre-exist or result from migraine experience predisposing to attack recurrence and producing pain maintenance and chronicity. The foregone implications in treatment regard both pharmacological and non-pharmacological approaches. In considering the efficacy of drugs on migraine severity, the effects on the behavioural aspects should be taken into account. In acute therapy, the central effects of triptans should modify the pain pathway functions, although the clinical efficacy seems to be linked mostly with the action at vascular levels [29]. In migraine prophylaxis, the drugs with proved efficacy on attack recurrence, such as amitriptyline, propranol and valproate, exert effects on anxiety and mood disorders. In this view, the non-pharmacological approach to migraine appears apposite: relaxation/biofeedback training was as efficacious as propranol in preventing migraine [30]. In our opinion, a complete management of migraine patients needs the psychophysiologic aspects to be well known, according to the most recent reports [31]. The opportunity of biobehavioural management is well known in tension-type headache for the high comorbidity with psychiatric disorders [32, 33] and these primary headaches may share the same approach, particularly in the chronic form.

"To consider the sensory features of pain, and ignore its motivation and affective properties, is to look at only part of the problem, and not even the most important part at that" [34]: this has to be well deserved in every pain-related syndrome including migraine.

\section{References}

1. Ferrari MD, Haan J (2001) Genetics of headache. In: Silberstein SD, Lipton RB, Dalessio DJ (eds) Wollf's headache and other head pain. Oxford University, New York, pp 73-84

2. Goadbsy PJ, Edvinsson L, Ekman R (1990) Vasoactive peptide release in the extracerebral circulation of humans during migraine headache. Ann Neurol 28:183-187

3. Moskowitz MA (1990) Basic mechanisms in vascular headache. Neurol Clin 8:801-815

4. Connolly JF, Gawel M, Rose FC (1982) Migraine patients exhibit abnormalities in the visual evoked potentials. J Neurol Neurosurg Psychiatry 45:464-467

5. Puca FM, de Tommaso M, Savarese MA, Genco S, Prudenzano A (1992) Topographic analysis of steady-state visual evoked potentials (SVEPs) in the medium frequency range in migraine with and without aura. Cephalalgia 12:245-249
6. Bocker KBE, Timsit-Berthier M, Timisit M (1990) Contingent negative variation $(\mathrm{CNV})$ in migraine. Headache 30:604-609

7. Kropp P, Gerber WD (1993) Is increased amplitude of contingent negative variation in migraine due to cortical hyperactivity or to reduced habituation? Cephalalgia 13:37-41

8. Wang W, Timsit-Berthier M, Shoenen J (1996) Intensity dependence of auditory evoked potentials is pronounced in migraine: an indication of cortical potentiation and low serotonergic neurotransmission? Neurology 46:1404-1409

9. Thomson RF, Berry SD, Rinaldi PC, Berger TW (1979) Habituation and orienting reflex: the dural process theory revised. In: Kimmel HD, van Olst EH, Orlebeke JF (eds) The orienting reflex in humans. Lawrence Erlbaum, Hillsdale, pp 21-60
10. Gottfries CG, von Knorring L, Perries C (1976) Neurophysiological measures related to levels of 5-hydroxyindoleacetic acid, homovanillic acid and tryptophan in cerebrospinal fluid of psychiatric patients.

Neuropsychobiology 2:1-8

11. Hegerl U, Juckel G (1993) Intensity dependence of auditory evoked potentials as an indicator of central serotoninergic neurotransmission: a new hypothesis. Biol Psychiatry 33:173-187

12. Goadbsy PJ (2000) The pharmacology of headache. Prog Neurobiol 62(5):509-525

13. Wang W, Wang GP, Ding XL, Wang YH (1999) Personality and response to repeated visual stimulation in migraine and tension-type headache. Cephalalgia 19(8):718-724

14. de Tommaso M, Murasecco D, Libro G, Guido M, Sciruicchio V, Di Fruscolo O, Monetti C, Gallai V, Puca F (2001)

Modulation of trigeminal reflex excitability in migraine: effects of attention and habituation on the blink reflex. Int J Psychophysiol (in press) 
15. Buchsbaum MS, Goodwin F, Murhy D, Borge G (1971) AER in affective disorders. Am J Psychiatry 128:51-57

16. Hegerl U, Herrman WM (1990) Eventrelated potentials and the prediction of differential drugs response in psychiatry. Neuropsychobiology 23:99-108

17. Breslau N, Schultz LR, Stewart WF, Lipton R, Welch KM (2001) Headache types and panic disorder: directionality and specificity. Neurology 56(3):350-354

18. Lipton RB, Hamelsky SW, Kolodner KB, Steiner TJ, Stewart WF (2000) Migraine, quality of life, and depression: a population-based case-control study. Neurology 55(5):629-635

19. Juang KD, Wang SJ, Fuh JL, Lu SR, Su TP (2000) Comorbidity of depressive and anxiety disorders in chronic daily headache and its subtypes. Headache 40:818-823

20. Guidetti V, Galli F, Fabrizi P, Giannantoni AS, Napoli L, Bruni O, Trillo S (1998) Headache and psychiatric comorbidity: clinical aspects and outcome in an 8-year follow-up study. Cephalalgia 18(7):455-462

21. Genco S, de Tommaso M, Prudenzano AM, Savarese M, Puca FM (1994) EEG features in juvenile migraine: topographic analysis of spontaneous and visual evoked brain electrical activity: a comparison with adult migraine. Cephalalgia 14(1):41-46
22. de Tommaso M, Guido M, Libro G, Sciruicchio V, Puca F (2000) The three responses of the blink reflex in adult and juvenile migraine. Acta Neurol Belg 100(2):96-102

23. Fields HL (2000) Pain modulation: expectation, opioid analgesia and virtual pain. Prog Brain Res 122:245-253

24. Burstein R (2001) Deconstructing migraine headache into peripheral and central sensitization. Pain 89(2-3):107-110

25. Fusco BM, Colantoni O, Giacovazzo M (1997) Alteration of central excitation circuits in chronic headache and analgesic misuse. Headache 37(8):486-491

26. de Tommaso M, Puca FM (2001)

L'elaborazione del dolore nelle cefalee croniche: potenziali evocati da stimolo nocicettivo nella emicrania cronicizzata. In: Atti del $\mathrm{XV}^{\circ}$ Congresso SISC, Florence, 10-12 June 2001

27. Flor $\mathrm{H}$ (2000) The functional organization of the brain in chronic pain. Prog Brain Res 129:313-322

28. Buchner H, Reinartz U, Waberski TD, Gobbele R, Noppeney U, Scherg M (1999) Sustained attention modulates the immediate effect of de-afferentation on the cortical representation of the digits: source localization of somatosensory evoked potentials in humans. Neurosci Lett 260(1):57-60
29. de Tommaso M, Guido M, Libro G, Sciruicchio V, Puca F (2000)

Zolmitriptan reverses blink reflex changes induced during the migraine attack in humans. Neurosci Lett 289(1):57-60

30. Holroyd KA, Penzien DB (1990) Pharmacological versus non-pharmacological prophylaxis of recurrent migraine headache: a meta-analytic review of clinical trials. Pain 42(1):1-13

31. Massiou H (2000) Prophylactic treatments of migraine. Rev Neurol 156[Suppl 4]:S79-S86

32. Puca F, Genco S, Prudenzano MP et al (1999) Psychiatric comorbidity and psychosocial stress in patients with tension-type headache from headache centers in Italy. The Italian Collaborative Group for the Study of Psychopathological Factors in Primary Headaches. Cephalalgia 19(3):159-164

33. Holroyd KA, O'Donnell FJ, Stensland M, Lipchik GL, Cordingley GE, Carlson BW (2001) Management of chronic tension-type headache with tricyclic antidepressant medication, stress management therapy, and their combination: a randomized controlled trial JAMA 285(17):2208-2215

34. Melzack R, Casey KL (1967) Localized temperature changes evoked in the brain by somatic stimulation. Exp Neurol 17(3):276-292 